\title{
Prevalencia de enfermedades de transmisión sexual en pacientes con VIH/SIDA
}

\section{Prevalence of sexually transmitted diseases in HIVIAIDS patients}

\author{
Carmen G. Santamaría-Burgos*, Carlos E. Oliva-Torres, Catherin R. Molina-Valencia, \\ Jefferson X. Molina-Quimbita y Melanie M. Noriega-Moreno \\ Unidad de Atención Integral de personas viviendo con VIH/SIDA, Hospital General Enrique Garcés, Quito, Ecuador
}

\begin{abstract}
Resumen
Antecedentes: Las enfermedades de transmisión sexual (ETS) son un conjunto de enfermedades infecciosas que se transmiten a través de relaciones sexuales. Las ETS y el VIH mantienen una estrecha relación; la infección por VIH incrementa la probabilidad de contraer una ETS y viceversa. Objetivos: Determinar las prevalencias de las enfermedades de transmisión sexual en pacientes con VIH/sida acorde a la identidad sexual, cuantificación de CD4 y su correlación estadística; e identificar la relación entre las enfermedades de transmisión sexual y el contacto con trabajadores sexuales en pacientes con VIH. Materiales y métodos: Estudio epidemiológico analítico retrospectivo en el cual se analizaron todas las historias clínicas de los pacientes positivos a VIH $(n=1,186)$, de los cuales 164 tuvieron ETS. Resultados: De 1,186 pacientes con VIH, 164 mostraron ETS y la sifilis tuvo la mayor prevalencia en todos los grupos de identidad sexual, sobre todo en hombres que tienen sexo con hombres (HSH). El mayor número de casos de ETS se observó en el grupo con un conteo de CD4 de 200 a $500 \mathrm{cel} / \mu \mathrm{L}$, con una relación estadísticamente significativa. De los pacientes que tuvieron una o más ETS, 53 indicaron relaciones con trabajadores sexuales y 111 pacientes lo negaron, sin relación estadísticamente significativa. Conclusiones: Los hombres que tienen sexo con hombres poseen la mayor cantidad de ETS, tal vez debido al mayor riesgo relacionado con las relaciones sexuales anogenitales; se observó que las verrugas anogenitales podían ocurrir con un recuento normal de CD4, en concordancia con otros estudios; la gonorrea se encuentra con cifras elevadas de CD4, pero la prevalencia de casos es insuficiente para evidenciar una generalización significativa.
\end{abstract}

Palabras clave: VIH. ETS. Sifilis. HSH. Trabajadores sexuales.

\section{Abstract}

Background: Sexually transmitted diseases (STDs) are a group of infectious diseases that are transmitted through sexual intercourse. STDs and HIV affect a close relationship, HIV infection increases the probability of contracting an STD and vice versa. Objectives: To determine the prevalence of sexually transmitted diseases in HIV / AIDS patients according to sexual identity, quantification of CD4 and its statistical correlation; identify the relationship between sexually transmitted diseases and contact with sex workers in HIV patients. Materials and methods: Retrospective analytical epidemiological study in the analysis of all the medical records of HIV positive patients $(n=1186)$, of the analyzes $(n=164)$ STDs. Results: Of 1186 HIV patients, 164 STD patients, the syphilis being the most prevalent in all sexual identity groups, mainly in men who have sex with men (MSM). The highest number of STD cases was identified in the group with a CD4 content of 200-500 cel / $\mu \mathrm{L}$, showing a statistically significant relationship. Of the patients considering an STD or more, 53 reported having had relations-

Correspondencia:

*Carmen G. Santamaría-Burgos

E-mail: c_santamaria2000@yahoo.es DOI: 10.24875/MCUT.200000 licencia CC BY-NC-ND (http://creativecommons.org/licenses/by-nc-nd/4.0/). 
hips with sex workers and 111 patients who denied, with no statistically significant relationship. Conclusions: Men who have sex with men have the highest amount of STDs, possibly due to the higher risk associated with anogenital sexual relations; Anogenital warts were found to have a normal CD4 count, consistent with other studies; Gonorrhea is found with high levels of CD4, however, the prevalence of cases is decreased to show significant generalization.

Key words: HIV. STD. Syphilis. MSM. Sex workers.

\section{Introducción}

Las enfermedades de transmisión sexual (ETS) son un conjunto de trastornos infecciosos con expresión clínica diferente. Se transmiten sobre todo a través de relaciones sexuales sin protección: vaginales, anales y orales; y la persona infectada puede o no mostrar síntomas ${ }^{1}$. De acuerdo con la Organización Mundial de la Salud (OMS), más de 1 millón de personas contraen ETS al día y la mayoría de los casos es asintomática o tiene síntomas leves que no permiten establecer un diagnóstico preciso; además, se calcula que al año unos 357 millones de individuos contraen algún tipo de ETS, como clamidia, gonorrea, sífilis o tricomoniasis ${ }^{2}$.

En la actualidad, el VIH es uno de los principales problemas de salud pública mundial. Según la OMS, a finales del año 2016 había alrededor de 36.7 millones de personas infectadas en el mundo y 1.8 millones de nuevas infecciones ${ }^{3}$.

Las ETS y el VIH mantienen una estrecha relación; la infección por VIH incrementa la probabilidad de contraer una ETS y viceversa, y las manifestaciones clínicas de las ETS se vuelven inespecíficas al experimentar una atenuación del sistema inmunitario y tienden a ser lesiones de mayor tamaño, repetitivas o persistentes ${ }^{4}$. Se calcula que un porcentaje elevado de pacientes con $\mathrm{VIH} /$ sida desconoce que puede contraer nuevas ETS, ya que no las reconoce clínicamente y los métodos diagnósticos y la vigilancia epidemiológica no son efectivos o son inexistentes en ciertos países ${ }^{5}$.

En Uganda se evaluó a 355 pacientes con $\mathrm{VIH}$, de los cuales el $21.4 \%$ mostró un diagnóstico clínico y microbiológico de ETS: sífilis, hepatitis $B$, herpes genital, condiloma acuminado, uretritis no gonocócica y gonorrea ${ }^{6}$. En el estudio realizado en Indonesia ${ }^{4}$ se valoró a 3,350 infectados con $\mathrm{VIH}$ y el $4.2 \%$ tuvo una ETS; las de mayor prevalencia fueron condiloma acuminado, úlceras genitales inespecíficas, sífilis, herpes genital y sarna.

En El Salvador se revisaron 1,703 historias clínicas de pacientes con $\mathrm{VIH} /$ sida y en ellas predominó la sífilis con 171 casos, con una mayor frecuencia en pacientes masculinos y $83 \%$ de los cuales correspondió a hombres que tenían sexo con hombres ${ }^{7}$. En Argentina ${ }^{8}$ se identificó a 87 pacientes positivos a VIH en clínicas y hospitales y 52 registraron coinfección con sífilis.

En la región costera de Ecuador ${ }^{9}$ se valoró a una población de 5,988 mujeres que tuvieron trabajo de parto o aborto espontáneo, con una prevalencia de $\mathrm{VIH}$ y sífilis que corresponde a $1.13 \%$ y $0.37 \%$, respectivamente, lo que revela que la prevalencia de $\mathrm{VIH}$ excede el umbral que define a una epidemia generalizada.

En Ecuador la tasa de prevalencia de $\mathrm{VIH}$ en 2017 fue de $0.3 \%$ en el grupo de 15 a 49 años de edad, considerado como población sexualmente activa. Las provincias con mayor concentración de casos nuevos $(3,533)$ fueron Guayas $(31 \%)$, Pichincha $(23 \%)$, Esmeraldas (7\%), El Oro (5\%), Los Ríos y Manabí $(4.9 \%)^{10}$. En la actualidad, Ecuador posee 48 unidades de Atención Integral de VIH, de las cuales el Ministerio de Salud Pública (MSP) tiene 40, el Instituto Ecuatoriano de Seguridad Social (IESS) 3, las Fuerzas Armadas 2 y la Policía Nacional $2^{10}$.

La sífilis muestra mayor incidencia en pacientes coinfectados por VIH, en particular en la población de hombres que tienen sexo con hombres ${ }^{11}$. Los objetivos de este trabajo fueron determinar las prevalencias de las enfermedades de transmisión sexual en pacientes con $\mathrm{VIH} /$ sida de acuerdo con la identidad sexual, cuantificación de CD4 y correlación estadística; e identificar la relación entre las enfermedades de transmisión sexual y el contacto con trabajadores sexuales en pacientes con $\mathrm{VIH}$.

\section{Materiales y métodos}

Se llevó a cabo un estudio de diseño epidemiológico analítico retrospectivo en el cual se revisaron todas las historias clínicas de los pacientes positivos a VIH ( $n=1,186)$, de los cuales 164 desarrollaron enfermedades de transmisión sexual, sustentadas en diagnósticos clínicos, de laboratorio y microbiológicos.

El Hospital General Enrique Garcés es el tercer hospital general más importante de la ciudad de Quito y pertenece al Ministerio de Salud Pública y acoge cada semana a 3,000 pacientes en consulta externa; parte de ésta es la Unidad de Atención Integral de personas viviendo con VIH/SIDA, cuya consulta y medicación 
Tabla 1. Prevalencia de enfermedades de transmisión sexual en relación con el contacto con trabajadores sexuales

\begin{tabular}{|l|c|c|c|c|}
\hline \multirow{2}{*}{ ETS } & \multicolumn{3}{|c|}{ Contacto sexual } \\
\cline { 2 - 5 } & Número de casos & Sí & No & No responde \\
\hline Sífilis & 116 & 19 & 80 & 17 \\
\hline Verrugas anogenitales & 27 & 7 & 17 & 3 \\
\hline Candidiasis genital & 6 & 2 & 4 & 0 \\
\hline Herpes genital & 10 & 3 & 6 & 1 \\
\hline Donovanosis & 3 & 1 & 2 & 0 \\
\hline Gonorrea & 2 & 0 & 2 & 0 \\
\hline Total & 164 & 53 & 111 & 21 \\
\hline
\end{tabular}

son gratuitas cualesquiera que sean el estrato económico o la nacionalidad.

Con base en las historias clínicas de la Unidad de Atención Integral de personas viviendo con VIH/SIDA del Hospital Enrique Garcés, entre enero de 2016 y diciembre de 2017 se recogieron las siguientes variables: incidencia de las ETS, conteo de CD4, grupo de edad, identidad sexual, sexo, estadio clínico de $\mathrm{VIH}$ al diagnóstico de la ETS, contacto con trabajadores sexuales (hombres o mujeres).

Los criterios de inclusión fueron: historias clínicas de los pacientes que poseen un diagnóstico de VIH confirmado por ELISA o Western Blot coinfectados con una ETS de enero de 2016 a diciembre de 2017, nacionales y extranjeros de todas las edades, identidad sexual y sexo. Además, se incluyó a los pacientes con VIH coinfectados con sífilis que tuvieran estudios inmunológicos o no inmunológicos positivos. Para el cálculo de prevalencias se excluyeron todas las historias clínicas que carecieran de estudio de recuento de valores de linfocitos CD4.

El cálculo estadístico se realizó en el programa IBPSPSS statistics versión 25 del año 2017. Se ejecutaron pruebas estadísticas que son específicas para este diseño: $\chi^{2}$. La significancia estadística fue una $p<0.05$. Para el análisis estadístico entre el conteo de CD4 y las prevalencias de ETS se descartaron la gonorrea y donovanosis puesto que no se contó con una muestra significativa, pero se consideraron para determinar su prevalencia en pacientes con VIH.

\section{Resultados}

La prevalencia de ETS en el grupo estudiado fue de $13.82 \%$ ( $n=164)$ de las 1,186 historias clínicas de pacientes infectados con VIH. La población de hombres fue de 899 pacientes, de los cuales el 17.3\% $(n=156)$ tuvo una ETS y 287 pacientes fueron mujeres con una prevalencia de ETS de $2.7 \%(n=8)$.

El $34.23 \%$ de los pacientes $(n=406)$ manifestó haber mantenido relaciones con trabajadores sexuales con uso eventual de protección; los datos registrados no determinan el número de parejas sexuales y tipo de relación; se debe recalcar la dificultad para la obtención de estos datos debido a la subjetividad adjunta, dado que se encuentra en dependencia directa de encuestas realizadas a los pacientes y por tanto compromete la fiabilidad de la información. De los pacientes que tuvieron una o más ETS, 53 señalaron haber mantenido relaciones con trabajadores sexuales y 111 pacientes negaron su relación (Tabla 1). El análisis estadístico no muestra una relación significativa entre la presencia de ETS y el contacto con trabajadores sexuales $(p=0.86)$.

La edad mínima de la población estudiada fue de 17 y la máxima de 81 años; el grupo de edad predominante fue el adulto joven, de 18 a 35 años ( $n=97)$, seguido del adulto de 36 a 64 años $(n=63)$, adulto mayor de 65 años $(n=3)$ y adolescente de 14 a 17 años $(n=1)$. En la distribución por sexo predomina el masculino con un $95.7 \%(n=156)$ y el femenino con $4.3 \%(n=8)$. La proporción de HSH fue la de mayor presencia entre los casos de ETS estudiados ( $n=61)$, seguido de identidad heterosexual $(n=52)$ y bisexual ( $n=51$ ).

De acuerdo con las fases clínicas de los pacientes con VIH/sida que tuvieron una infección por ETS: $90 \%$ $(n=148)$ de pacientes en fase II, 3.65\% $(n=6)$ en fase III y $6.09 \%(n=10)$ en fase IV. 
Tabla 2. Prevalencia de enfermedades de transmisión sexual relacionadas con la identidad sexual

\begin{tabular}{|l|c|c|c|c|}
\multirow{2}{*}{ ETS } & \multicolumn{3}{|c|}{ Identidad sexual } & Total \\
\cline { 2 - 4 } & Heterosexual & HSH & Bisexual & $116(70.7 \%)$ \\
\hline Sífilis & $34(65.4 \%)$ & $45(73.8 \%)$ & $37(72.5 \%)$ & $27(16.5 \%)$ \\
\hline Verrugas anogenitales & $8(15.4 \%)$ & $9(14.8 \%)$ & $10(19.6 \%)$ & $6(3.7 \%)$ \\
\hline Candidiasis genital & $4(7.7 \%)$ & $0(0 \%)$ & $2(3.9 \%)$ & $10(6.1 \%)$ \\
\hline Herpes genital & $5(9.6 \%)$ & $3(4.9 \%)$ & $2(3.9 \%)$ & $3(1.8 \%)$ \\
\hline Donovanosis & $1(1.9 \%)$ & $2(3.3 \%)$ & $0(0 \%)$ & $2(1.2 \%)$ \\
\hline Gonorrea & $0(0 \%)$ & $2(3.3 \%)$ & $0(0 \%)$ & $164(100 \%)$ \\
\hline Total & $52(100 \%)$ & $61(100 \%)$ & $51(100 \%)$ & \\
\hline
\end{tabular}

Tabla 3. Prevalencia de enfermedades de transmisión sexual relacionadas con conteo de CD4

\begin{tabular}{|l|c|c|c|c|c|}
\hline CD4 & Sífilis n/\% & Verrugas anogenitales n/\% & Candidiasis genital $n / \%$ & Herpes genital n/\% & Total \\
\hline$<200$ & $26(22.4 \%)$ & $10(37 \%)$ & $1(16.7 \%)$ & $3(30 \%)$ & $40(25.1 \%)$ \\
\hline $200-500$ & $54(46.6 \%)$ & $10(37 \%)$ & $2(33.3 \%)$ & $5(50 \%)$ & $71(44.6 \%)$ \\
\hline$>500$ & $36(31 \%)$ & $7(25.9 \%)$ & $3(50 \%)$ & $2(20 \%)$ & $48(30.1 \%)$ \\
\hline Total & $116(100 \%)$ & $27(100 \%)$ & $6(100 \%)$ & $10(100 \%)$ & $159(100 \%)$ \\
\hline
\end{tabular}

La prevalencia de sífilis es predominante en todos los grupos de identidad sexual, con un mayor porcentaje en los pacientes HSH con una prevalencia de $73.8 \%$; las verrugas anogenitales con un $19.6 \%$ predominan en el grupo bisexual; la candidiasis genital y el herpes genital en el grupo heterosexual; y la donovanosis y la gonorrea se presentaron con $3.3 \%$ en $\mathrm{HSH}$ (Tabla 2). El análisis estadístico muestra una relación significativa entre las prevalencias de ETS y la identidad sexual $(p=0.0142)$.

De acuerdo con la distribución de grupos divididos por conteo de linfocitos TCD4, se reconoce un mayor número de pacientes en el grupo de 200-500 cel/ $\mu \mathrm{L}$ $(n=71)$, seguido del grupo con conteo $>500 \mathrm{cel} / \mu \mathrm{L}$ ( $\mathrm{n}=48)$ y el de $<200 \mathrm{cel} / \mu \mathrm{L}(\mathrm{n}=40)$ de ETS. Se observa que en el grupo de pacientes con conteo de linfocitos TCD4 de 200-500 cel/ $\mu \mathrm{L}$ se registró un mayor porcentaje de sífilis $(n=54)$ y herpes genital $(n=5)$; en los grupos de conteo de $<200$ y 200-500 cel/ $\mu \mathrm{L}$ se encontró una mayor prevalencia de verrugas (Tabla 3); de acuerdo con estos resultados, la donovanosis mostró un conteo de linfocitos $<200 \mathrm{cel} / \mu \mathrm{L}$ y la gonorrea de $>500 \mathrm{cel} / \mu \mathrm{L}$ (Fig. 1). El análisis estadístico evidencia una relación significativa entre las prevalencias de ETS observadas y las agrupaciones de la muestra basadas en el conteo de linfocitos TCD4 ( $p=0.0001)$.

Respecto de las categorías de las ETS, se advierte que en toda la población estudiada la sífilis es la de mayor frecuencia $(n=116)$ con $9.7 \%$, seguida de las verrugas anogenitales $(n=27)$ con $2.2 \%$; herpes genital $(n=10)$ con $0.8 \%$; candidiasis genital $(n=6)$ con $0.5 \%$; donovanosis $(n=3)$ con $0.2 \%$; y gonorrea $(n=2)$ con $0.1 \%$ (Fig. 1).

\section{Discusión}

Se encontró que el $34.23 \%$ de los infectados con $\mathrm{VIH}$ tuvo contacto con trabajadores sexuales. De acuerdo con los Centers for Disease Control and Prevention $(\mathrm{CDC})$, las personas que comercian con relaciones sexuales poseen un riesgo mayor de contraer o transmitir el VIH y las ETS porque posiblemente tengan conductas sexuales de riesgo (relaciones sexuales con varias parejas o nulo uso de preservativo); sin embargo, a falta de estudios en la población de individuos que intercambian relaciones sexuales, existen grandes obstáculos para iniciativas de prevención ${ }^{12}$ y dificultan la obtención de datos fiables que permitan establecer 


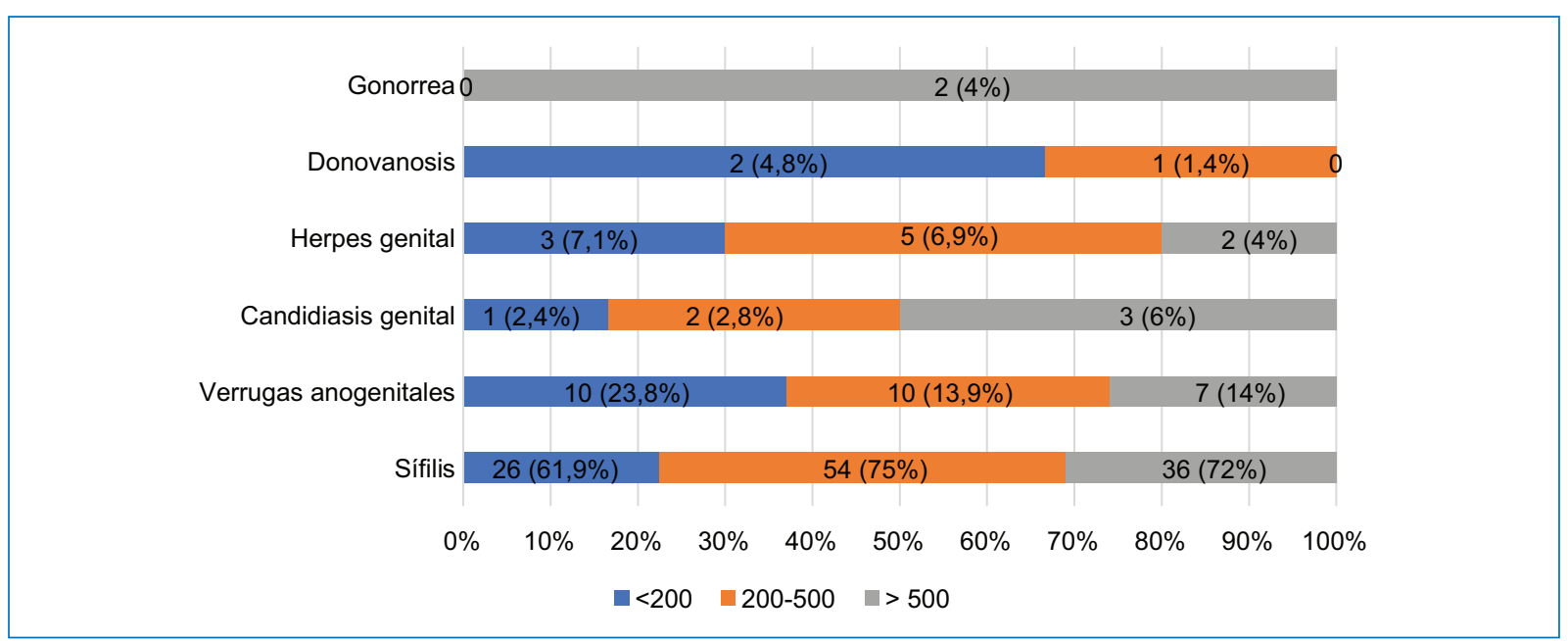

Figura 1. Conteo de linfocitos CD4 y su distribución de acuerdo con sus rangos en las enfermedades de transmisión sexual analizadas.

la relación objetiva entre la incidencia de ETS y el contacto con trabajadores sexuales.

La prevalencia de ETS en este estudio fue de $13.82 \%$ $(n=164)$, que se aparta de los datos de Katusiime ${ }^{6}$ con $21.4 \%(n=76)$, tal vez debido a que el tamaño de la muestra en ese estudio fue reducido (355 pacientes). Los resultados de Harnanti ${ }^{4}$, que registraron una prevalencia de $4.2 \%(n=148)$, con un tamaño de muestra de 3,350 pacientes, divergen del estudio de los autores quizá por la ubicación geográfica en Surubaya-Indonesia y las diferencias de las conductas sexuales.

De los pacientes con ETS, en la distribución por sexo predomina el masculino con un $95.7 \%(n=156)$, con el femenino sólo son $4.3 \%(n=8)$, y se observa una mayor prevalencia en hombres con $17.3 \%$ mientras que en la población femenina es de $2.7 \%$; no obstante, el tamaño de la muestra en mujeres es reducido, por lo que es recomendable realizar nuevos estudios respecto de la prevalencia de ETS en sujetos de sexo femenino, pese a lo cual los datos de prevalencia son similares a los que informó Katusiime ${ }^{6}$.

La edad de presentación corresponde a la sexualmente activa ( $n=97)$, similar al estudio de HuttonRose $^{13}$ en el que el grupo de edad más señalado fue de 30 a 34 años.

Los pacientes HSH tuvieron el mayor número de casos y prevalencia de ETS y en proporciones similares se encontraron las poblaciones bisexual y heterosexual, comparable a los datos de Ortega $^{7}$ y $\mathrm{Pech}^{14}$ en cuyo estudio fueron determinantes las dermatosis no infecciosas e infecciosas, incluidas las ETS. Sin embargo, el protocolo anterior difiere del estudio de
Prabhakaran ${ }^{15}$ en el que la mayor frecuencia corresponde al grupo heterosexual, seguido por el de HSH y el bisexual, posiblemente por una variación en la muestra $(n=170)$ y la ubicación geográfica en India. Los estudios de Aydin ${ }^{16}$ y Sud ${ }^{17}$ registraron una mayor frecuencia de heterosexuales, sobre todo porque valoraron la vía de transmisión. El análisis estadístico muestra una relación significativa entre las prevalencias de ETS y la identidad sexual. De acuerdo con los $\mathrm{CDC}^{18}$, las relaciones sexuales anales representan el mayor riesgo de transmisión de $\mathrm{VIH}$, puesto que la probabilidad de infección en la pareja receptiva es 13 veces mayor respecto de la pareja insertiva, debido en especial a que la mucosa del recto es delgada y permite el paso del VIH al cuerpo; además, pueden contraerse otras ETS como clamidia y gonorrea mediante relaciones sexuales anales sin preservativo y algunas pueden transmitirse por contacto piel con piel, entre ellas la sífilis o el herpes.

La sífilis es la ETS más frecuente identificada, con una prevalencia de $9.7 \%$ ( $n=116)$, en concordancia con los datos de Mayer ${ }^{19}$ con $6.4 \%$. Además, Motta ${ }^{20}$ informó una prevalencia de sífilis del $43.2 \%$ tal vez debido a un tamaño de muestra de 220 pacientes y se limitó a investigar las prevalencias de sífilis, herpes y condilomatosis (Fig. 2). En segundo lugar destacan las verrugas anogenitales con $2.2 \%$ que contrastan con los datos de Prabhakaran ${ }^{15}$ y Chopra ${ }^{21}$ con 2.4 y $7.7 \%$, respectivamente, que tuvieron una mayor prevalencia quizá porque determinaron manifestaciones infecciosas y no infecciosas y en virtud de la ubicación geográfica de la India. 


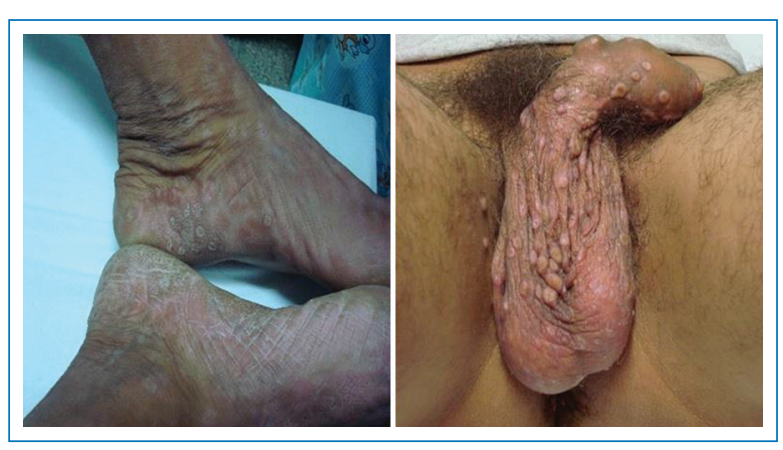

Figura 2. Sífilis secundaria: pueden observarse sifilides características como condilomas planos en genitales y sifílides papulosas en plantas de los pies de pacientes positivos a VIH.

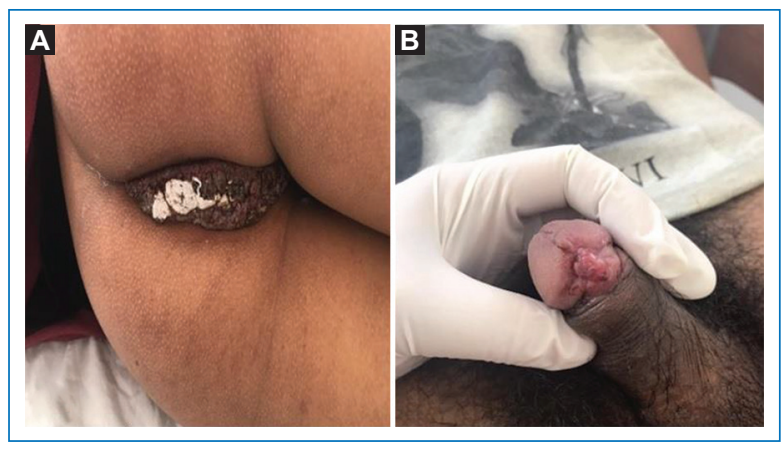

Figura 3. Infección por HPV. A: tumor de BuschkeLowenstein. B: condiloma acuminado en pacientes con VIH.

En los estudios de Sud ${ }^{17}$ y Titou ${ }^{22}$, la presentación de sífilis en pacientes con $\mathrm{VIH}$ se encuentra en el recuento de 200-500 cel/ $\mu \mathrm{L}$. En la investigación de Prabhakaran ${ }^{15}$, el condiloma acuminado fue la ETS más común con un recuento medio de CD4 de $320 \mathrm{cel} /$ $\mu \mathrm{L}$; Goldstein ${ }^{23}$ observó que el condiloma acuminado aparece con una inmunosupresión avanzada de CD4 $<75 \mathrm{cel} / \mu \mathrm{L}$, mientras que el estudio de $\mathrm{Kim}^{24}$ señaló que el condiloma acuminado es más frecuente en pacientes con recuento de CD4 $>200 \mathrm{cel} / \mu \mathrm{L}$. Los datos de los autores se equiparan a los anteriores y puede señalarse que esta infección puede ocurrir incluso con un recuento normal de CD4, pero en este estudio la población de muestra no es muy grande (Fig. 3).

El mayor número de casos de herpes genital $(n=7)$ se reconoció en el recuento de linfocitos CD4 >200, con ligera similitud al protocolo de Barbour ${ }^{25}$ que determinó que las personas infectadas en fecha reciente por VIH-1/VHS-2 obtuvieron mayores recuentos de TCD4

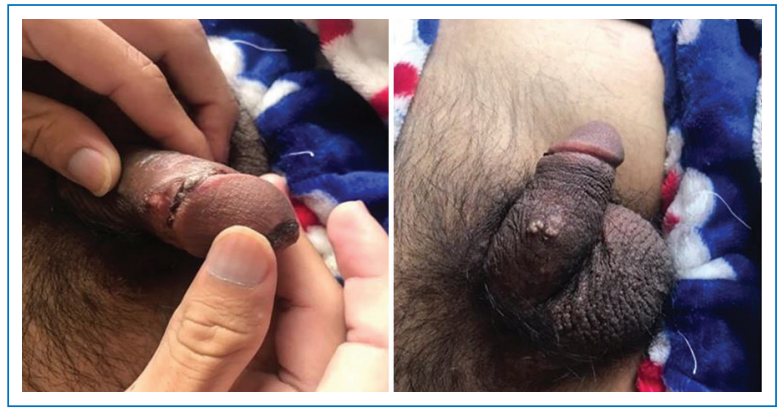

Figura 4. Infección por herpes genital en paciente con VIH.

con el tiempo que aquéllas sin coinfección; sin embargo, la infección por HVS después de la adquisición del VIH-1 no tuvo efecto en el recuento de CD4 (Fig. 4).

En estricto sentido, la donovanosis no se considera una enfermedad de transmisión sexual que afecta a individuos sexualmente activos ${ }^{26}$. Se ha identificado una relación entre la donovanosis y el $\mathrm{VIH}$, pero se desconoce si ésta eleva el riesgo de adquirir VIH. La coexistencia de las dos infecciones se manifiesta por úlceras persistentes y prolongadas, si bien el tamaño de éstas y la presentación clínica no son casi nunca diferentes respecto de quienes no tienen $\mathrm{VIH} .{ }^{27}$

Neisseria gonorrhoeae, causante de la gonorrea, libera un factor que induce la expresión de secuencias terminales repetidas largas (LTR) del VIH-1 en un grupo de diferenciación coinfectado de linfocitos $T$ positivos CD4, por lo que se vincula particularmente con la expresión del $\mathrm{VIH}-1^{28}$. El estudio de Ghassabi ${ }^{29}$ reveló una media mayor de linfocitos CD4 entre los pacientes infectados con gonorrea en comparación con los pacientes no infectados (368 \pm 238 vs $415 \pm 328)$, aunque la diferencia no fue estadísticamente significativa, similar a los resultados de los autores en dos casos con un recuento de linfocitos $\mathrm{CD} 4>500 \mathrm{cel} / \mu \mathrm{L}$.

Existen limitantes que pueden servir para conducir nuevas investigaciones, entre ellas la baja prevalencia de ETS para encontrar relaciones y generalizaciones significativas; la falta de evidencia científica de pacientes con VIH coinfectados con ETS y su relación con trabajadores sexuales; los datos autoinformados como el contacto con trabajadores sexuales, uso de métodos de protección y número de parejas sexuales; y el carácter retrospectivo de este estudio que limita la información a los datos ya recolectados, por lo cual es preciso desarrollar nuevos estudios prospectivos. 


\section{Conclusiones}

Se identificó una relación estadísticamente significativa entre las prevalencias de las enfermedades de transmisión sexual y la identidad sexual $(p=0.0142)$, lo cual demostró que los HSH tienen mayor cantidad de ETS debido con toda probabilidad a que la práctica de la relación sexual anogenital representa un mayor riesgo de contagio.

La sífilis prevaleció sobre todas las ETS y se reconoció una mayor prevalencia en el grupo de 200-500 cel/ $\mu \mathrm{L}$; las verrugas anogenitales en estudios de comparación podrían relacionarse con un recuento normal de CD4; de acuerdo con la evidencia, la gonorrea se acompaña de cifras elevadas de CD4, pero la prevalencia de los estudios se encuentra disminuida, lo que constituye una limitación para hallar relaciones y emitir generalizaciones significativas.

Correlacionar las ETS con el conteo de CD4 puede ayudar a proponer medidas preventivas especialmente con los grupos de riesgo como los HSH.

La población que tuvo relaciones con trabajadores sexuales corresponde al $34.23 \%$. No se identificó una relación estadística entre las ETS y el contacto con trabajadores sexuales, pero este dato está sujeto a la encuesta realizada a los pacientes, por lo que constituye un sesgo en la fiabilidad de los datos encontrados.

\section{Agradecimientos}

Los autores expresan su agradecimiento al personal del Hospital Enrique Garcés.

\section{Financiamiento}

La presente investigación no ha recibido ayudas específicas provenientes de instituciones del sector público, sector comercial o entidades sin ánimo de lucro.

\section{Conflicto de intereses}

Los autores declaran no tener ningún conflicto de intereses

\section{Responsabilidades éticas}

Protección de personas y animales. Los autores declaran que para esta investigación no se han realizado experimentos en seres humanos ni en animales.
Confidencialidad de los datos. Los autores declaran que han seguido los protocolos de su centro de trabajo sobre la publicación de datos de pacientes.

Derecho a la privacidad y consentimiento informado. Los autores han obtenido el consentimiento informado de los pacientes y/o sujetos referidos en el artículo. Este documento obra en poder del autor de correspondencia.

\section{Bibliografía}

1. Flores $\mathrm{E}$, Martínez M, Alonso M, Hernández L. Intervención educativa en adolescentes sobre conocimientosen la prevención de ITS/VIH-SIDA. Medimay. 2017;24(2):127

2. Organización Mundial de la Salud. OMS Internet. 2019 citado 18 de enero 2019. Disponible en: https://www.who.int/es/news-room/factsheets/detail/sexually-transmitted-infections-(stis)

3. Organización Mundial de la Salud. OMS Internet. 2018 citado 18 de enero 2019. Disponible en: https://www.who.int/es/news-room/fact-sheets/ detail/hiv-aids

4. Harnanti D, Hidayati A, Miftahussurur M. Concomitant sexually transmitted diseases in patients with diagnosed hiv/aids: a retrospective study. Afr J Infect Dis. 2018;12(1S):83-84.

5. Díez M, Díaz A. Infecciones de transmisión sexual: epidemiología y control. Rev Esp Sanid Penit. 2011;13:58

6. Katusiime C, Schlech W, Parkes-Ratanshi R, Sempa J, Kambugu A Characteristics of sexually transmitted infections among high-risk hiv-positive patients attending an urban clinic in Uganda. Journal of the International Association of Providers of AIDS Care. 2016;15(1):36.

7. Ortega R, Awad P. Sífilis en pacientes portadores de VIH o con SIDA en un servicio de enfermedades de transmisión sexual. Rev Chilena Dermatol. 2009;25(1):32.

8. Griemberg G. Alta prevalencia de coinfección por sífilis-VIH en cuatro hospitales de la ciudad de Buenos Aires, Argentina. Rev Argent Microbiol. 2006;38(3):134

9. Sánchez-Gómez A. HIV and syphilis infection in pregnant women in Ecuador: prevalence and characteristics of antenatal care HIV and syphilis infection in pregnant women in Ecuador: prevalence and characteristics of antenatal care. Sex Transm Infect. 2013;(0):1.

10. Ministerio de Salud Pública. MSP Internet. 2018 citado 18 de diciembre. Disponible en: https://www.salud.gob.ec/vih/

11. Prieto P, Imaza A, Calatayud L, García O, Saumoy M, Podzamczer D. Sífilis atípica extracutánea en pacientes con infección por VIH. Med Clin. 2017;149(11):2

12. Centro para el Control y Prevención de Enfermedades. CDC. Internet 2018. Citado 26 Feb 2018. Disponible en: https://www.cdc.gov/hiv/spanish/group/sexworkers.html

13. Hutton-Rose N, Blythe C, Ogbonna C. The prevalence of other sexually transmitted infections in confirmed HIV cases at a referral clinic in Jamaica. JR Soc Promotor de Salud. 2008;128(5):242.

14. Pech $L$, Pérez S, Peralta M. Manifestaciones dermatológicas en pacientes VIH y su correlación con la cantidad de linfocitos CD4 en la clínica de Infecciones de transmisión sexual del centro dermatológico. Dermatol Rev Mex. 2014;58:6-8.

15. Prabhakaran N, Telanseri J. Jaisankar, Hamide A, Malathi M, Kumari R Effect of antiretroviral therapy on mucocutaneous manifestations among human immunodeficiency virus-infected patients in a tertiary care centre in South India. Indian Journal of Sexually Transmitted Diseases and AIDS. 2015;36:4-8.

16. Aydin O, Karaosmanoğlu H, Korkusuz R, Özeren M, Nazlican O. Mucocutaneous manifestations and the relationship to CD4 lymphocyte counts among Turkish HIV/AIDS patients in ఐstanbul, Turkey. Turkish Journal of Medical Sciences. 2015;45:2-3.

17. Sud N, Shanker V, Sharma A, Sharma NL, Gupta M. Mucocutaneous manifestations in $150 \mathrm{HIV}$-infected Indian patients and their relationship with CD4 lymphocyte counts. International Journal of STD \& AIDS. 2009;20:772.

18. Centro para el Control y Prevención de Enfermedades. CDC. Internet 2016. Citado 26 Feb 2018. Disponible en: https://www.cdc.gov/hiv/spanish/risk/analsex.html

19. Mayer K. Which HIV-infected men who have sex with men in care are engaging in risky sex and acquiring sexually transmitted infections: findings from a Boston community health centre. Sex Transm Infect. 2010;86(1):66.

20. Motta A, Marín D, Merlo E. Prevalencia de herpes, sífilis y condilomatosis en pacientes con VIH en un hospital de referencia de Bogotá. Rev Asoc Colomb Dermatol. 2013;21(4):325.

21. Chopra S, Arora U. Skin and mucocutaneous manifestations: useful clinical predictors of HIV/AIDS. J Clin Diagn Res. 2012;6:1695. 
22. Titou $\mathrm{H}$, Ebongo $\mathrm{C}$, Hija N. Dermatologic manifestations among human. International Journal Dematology. 2017;57:2-3.

23. Goldstein B, Berman B, Sukenik E, Frankel SJ. Correlación de trastornos de la piel con recuentos de linfocitos CD4 en pacientes con VIH/SIDA. J Am Acad Dermatol. 1997;36:262.

24. Kim TG, Lee KH, Oh SH. Trastornos de la piel en pacientes coreanos infectados con el virus de la inmunodeficiencia humana y su asociación con un recuento de linfocitos CD4: un estudio preliminar. J Eur Acad Dermatol Venereol. 2010;24:1476.

25. Barbour J. HIV-1/HSV-2 coinfected adults in early HIV-1 infection have elevated CD4+T cell counts. PLoS one. 2007;2(10):2.
26. Pedroso M. Granuloma inguinal en paciente con VIH. Reporte de caso. Costa Rica. Rev Clin Esc Med. 2017;7(1):2

27. Gaviria-Sánchez V, Monterrosa-Castro A, Fernández-Daza E. Úlcera genital por Klebsiella granulomatis (donovanosis) en una pareja heterosexual. Rev Chil Obstet Ginecol. 2015;80(4):327.

28. Malott R. Neisseria gonorrhoeae-derived heptose elicits an innate immune response and drives HIV-1 expression. Proc Natl Acad Sci USA. 2013:110:10235

29. Ghassabi F. Gonorrhea and syphilis co-infection and related risk factors in HIV patients from Shiraz, South of Iran. Caspian J Intern Med. 2018;9(4):397 